\title{
Detailed Chemical Abundances in a Metal-Poor Stellar Stream
}

\author{
Ian U. Roederer, ${ }^{1}$ Christopher Sneden, ${ }^{1}$ Ian B. Thompson, ${ }^{2}$ \\ George W. Preston, ${ }^{2}$ and Stephen A. Shectman ${ }^{2}$ \\ ${ }^{1}$ Department of Astronomy, University of Texas at Austin, Austin, TX 78712 \\ email: iur@astro.as.utexas.edu \\ ${ }^{2}$ The Observatories of the Carnegie Institution of Washington, Pasadena, CA 91101
}

\begin{abstract}
We have observed 9 bright metal-poor stars whose kinematics suggest they are members of a stellar stream in the vicinity of the Solar neighborhood. These 9 stars exhibit no star-to-star dispersion in their $[\mathrm{X} / \mathrm{Fe}]$ ratios for the $\alpha$ and Fe-peak elements, and the neutroncapture elements suggest mild enrichment by the main $r$-process. The abundance patterns seen in this stream are very similar to those found in the metal-poor globular cluster M15, and the kinematics of M15 are similar to those of the stream, suggesting that these two groups of stars may have shared a common origin.
\end{abstract}

Keywords. Stars: abundances, stars: kinematics, stars: Population II, globular clusters: individual (M15), Galaxy: halo

Nearly a decade ago, Helmi et al. (1999) reported the detection of a group of low metallicity stars whose angular momentum components clumped together more than would be expected for a random distribution of halo stars. Members of this stellar stream are scattered throughout all parts of the sky, indistinguishable from "ordinary" halo stars, yet they are on very similar, eccentric orbits, indicating that the stars remember where they originated. We have obtained high resolution $(\mathrm{R} \sim 30,000-45,000)$ and high $\mathrm{S} / \mathrm{N}$ spectra of 9 members of this stream using the $2.7 \mathrm{~m}$ Smith Telescope at McDonald Observatory and the $6.5 \mathrm{~m}$ Magellan-Clay Telescope at Las Campanas Observatory. We have derived abundances for 20-30 elements in each star.

The $[\mathrm{C} / \mathrm{Fe}]$ ratios in these stars are subsolar $(\sim-0.3)$. The $[\alpha / \mathrm{Fe}]$ ratios are enhanced and typical of metal-poor halo stars, as are the Fe-peak species to Fe ratios. In all cases, there is no star-to-star dispersion within the uncertainties. The neutron-capture elements show clear signatures of enrichment by the main component of the rapid $(r)$ neutroncapture process for $Z \geqslant 62(-0.2<[\mathrm{Eu} / \mathrm{Fe}]<+0.5)$, while $\mathrm{Ba}$ to $\mathrm{Nd}$ show very mild overabundances relative to the scaled-solar $r$-process. Sr, Y, Zr, and Mo show somewhat higher overabundances. This pattern is found in all stars in the sample. The $[\mathrm{Pb} / \mathrm{Fe}]$ ratio is not enhanced as would be expected for enrichment from the slow $(s)$ neutron-capture process at low metallicity; this, together with the subsolar $[\mathrm{C} / \mathrm{Fe}]$ ratios, suggests that the excess of $\mathrm{Ba}-\mathrm{Nd}$ is not due to the $s$-process. The increasing excess relative to the main $r$ process with decreasing $Z$ for the neutron-capture elements, shown in Figure 1, suggests that the weak component of the $r$-process (e.g., HD 122563 as an empirical example of this enrichment pattern; Honda et al. 2007) may contribute to these elements. There is no star-to-star dispersion for the metal-rich stars in the sample $(-2.2<[\mathrm{Fe} / \mathrm{H}]<-1.5)$, but the two most metal-poor stars $(-2.5<[\mathrm{Fe} / \mathrm{H}]<-2.3)$ have somewhat lower levels of neutron-capture enrichment.

The overall chemical homogeneity - much more than the rest of the stellar halosuggests that these stars do share a common origin. Also, these enrichment patterns are 
not characteristic of any of the known Milky Way dSph or uFd systems. The globular cluster M15 $([\mathrm{Fe} / \mathrm{H}]=-2.6$; Sobeck et al. 2009$)$ has similar kinematic properties to the stars in the stream. This cluster has a wide dispersion of enrichment by the main $r$ process $(+0.2<[\mathrm{Eu} / \mathrm{Fe}]<+1.0)$ at a single metallicity, which is a unique characteristic among globular clusters. The level of $r$-process enrichment in M15 is also very similar to that found in the stream, with a total dispersion of order 1 dex occurring around a metallicity of $[\mathrm{Fe} / \mathrm{H}] \sim-2.5$ in both groups of stars. Figure 1 shows the neutron-capture abundances in the stream compared with those from three stars in M15; the abundances are identical for every element in common. The lighter elements in M15 stars are also very similar to the stars in the stream. The combination of kinematic and abundance similarities is evidence that these groups of stars may in fact share a common origin.

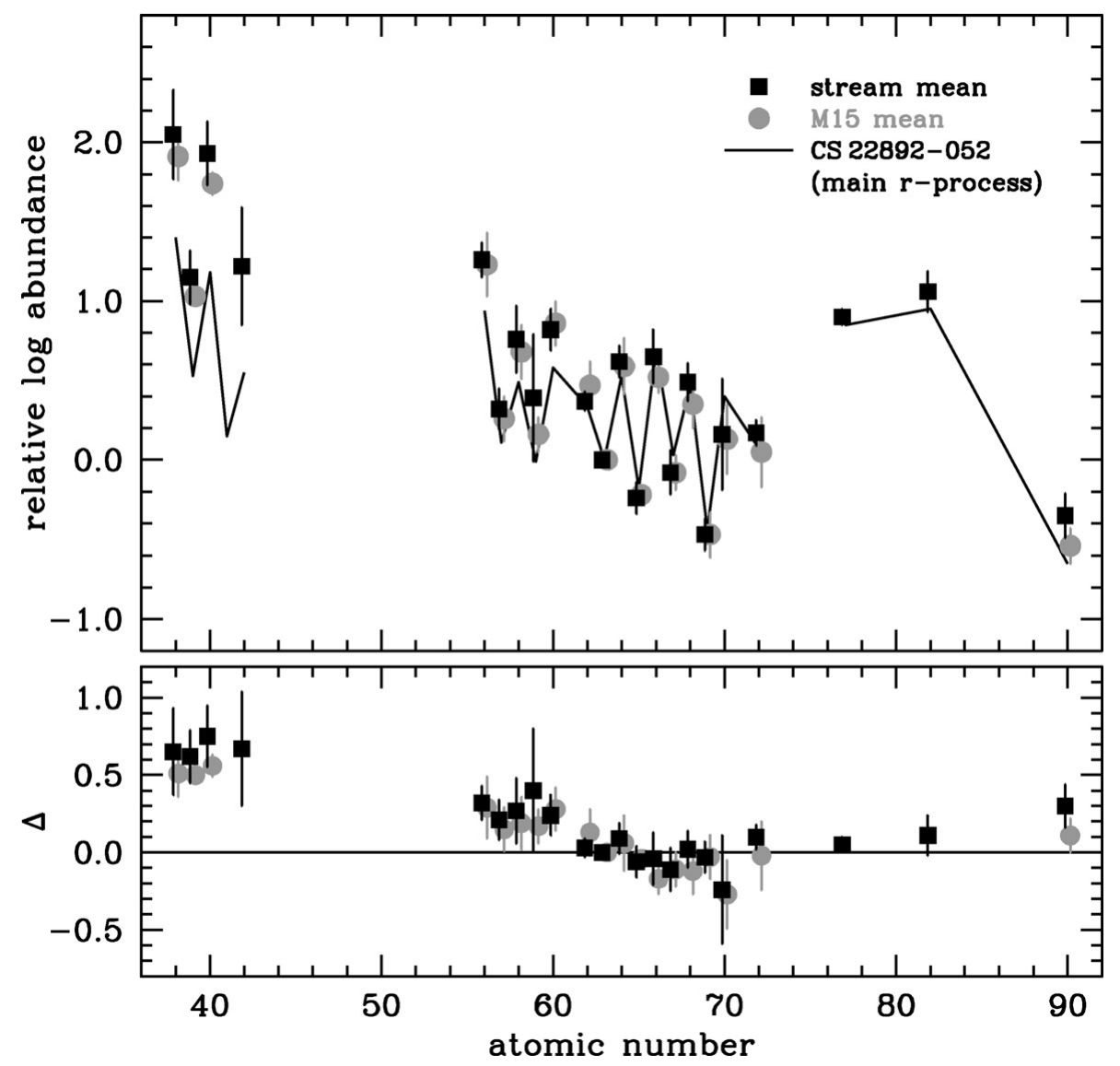

Figure 1. Comparison of the mean abundances in the stream stars (squares) and the globular cluster M15 (circles). CS 22892-052, which is strongly enriched by the main $r$-process (Sneden et al. 2009), is illustated by the line. All abundances are normalized at Eu.

\section{References}

Helmi, A., White, S. D. M., de Zeeuw, P. T., \& Zhao, H. 1999, Nature, 204, 53

Honda, S., Aoki, W., Ishimaru, Y., \& Wanajo, S. 2007, ApJ, 666, 1189

Sneden, C., Lawler, J. E., Cowan, J. J., Ivans, I. I., \& Den Hartog, E. A. 2009, ApJS, 182, 80

Sobeck, J. S. et al. 2009, in prep. 